\title{
Online employment portal architecture based on expert system
}

\author{
Janampally Himabindu Priyanka, Nikhat Parveen \\ Department of Computer Science and Engineering, Koneru Lakshmaiah Education Foundation, Vaddeswaram, Guntur, \\ Andhra Pradesh, India
}

\begin{tabular}{|c|c|}
\hline Article Info & ABSTRACT \\
\hline Article history: & \multirow{5}{*}{$\begin{array}{l}\text { Now a days, choosing the skill set which belongs to current marketing trends } \\
\text { that suits him/her is difficult especial for the fresher (who is an employee). It } \\
\text { is even more complex for the employer to requite the required skilled } \\
\text { person. As there will be huge data. To match these two requirements, we } \\
\text { need an expert system. An expert system which can cluster the data as well } \\
\text { as to answer the query posted by both employee and employer. In this paper, } \\
\text { component-based architecture is described which includes cloud computing, } \\
\text { the cluster and software agents. }\end{array}$} \\
\hline Received Jul 28, 2021 & \\
\hline Revised Dec 24, 2021 & \\
\hline Accepted Jan 13, 2022 & \\
\hline Keywords: & \\
\hline
\end{tabular}

Agent based system

Clustering

Component based architecture

Expert system

Software agents

This is an open access article under the CC BY-SA license.

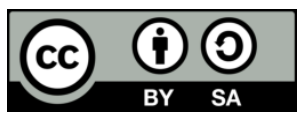

\section{Corresponding Author:}

Janampally Himabindu Priyanka

Department of Computer Science and Engineering, Koneru Lakshmaiah Education Foundation

Vaddeswaram, Guntur, Andhra Pradesh, India

Email: himabindupriyanka1@gmail.com

\section{INTRODUCTION}

In the current situation, online job portals have begun to receive a large number of resumes in various styles and designs from job seekers with a variety of educational backgrounds, work experiences, and skills [1], [2]. Finding and hiring the proper talent from a diverse pool of candidates is one of the most difficult and time-consuming tasks for any company's human resource department (HRD) [3], [4]. To meet this challenge, a number of companies have turned to e-selecting phases [5], [6]. According to, there were over 40,000 e-enlistment sites in 2012 to assist job seekers and scouts all around the world. According to the International Association of Employment Web Sites (IAEWS) [7], there are more than 60,000 e-enlistment frameworks in use in 2017. Fortunately, randomised studies provide a more robust technique for detecting causal companion impacts in networks [8]-[10].

To deal with such type of data one should use big data. Utilizing enormous information data is being made at an outstandingly fast speed and coming to fruition into a massive data, data shows different features, for instance, colossal volume, different collection, incredibly evolving, multivalued, fluctuating snappiness [11], conventional data clustering techniques make it harder to examine the data and recover the information. Extract the necessary information and afterward find the new information by the disguised association between data is called information mining. The various stages in which data mining is handled, [12], [13] which helps the business relationship to support their business and supporting them in unique.

To get the fast outcome from such tremendous information. A programme that runs without the aid of people and some extra programming is required. Prior to going any further, it is critical to define the significance of an agent-based system [14]. This is the fundamental topic of thought that is employed by the administrator although expert-based structures may include a thoughtful consideration professional (such as 
UI administrators or programmer secretaries [15]), the usage of an agent-based system appears to be the most optimal [16]. The structure that likes to go with qualities [17], according to a subject matter expert:

Autonomy refers to the ability of professionals to collaborate without the aid of people or other systems.

Reactivity: The ability of experts to alter the atmosphere and remake themselves at any moment.

Pro-animation: The ability to adapt to changes in behavior.

- Social capacity: The ability to receive and deliver signals via expert language among various professionals (and maybe humans).

In [18], [19] the creators talked about three new techniques that are utilized in grouping informational collections. The method utilized was gradual k-means where clustering was utilizing math information. Improved k-modes algorithm the groups were used clear information; while on account of blended math and clear figures, k-prototype is definite. Sreeram [20] discussed worldwide plan for development to reduce the technological problems. The technique uses selection loop but needs identical aptitudes. As sequence of importance waits the datasets are appropriated into a few groups. Headdown/structure up operation, both of them can be used for different stages of clustering. Top-down structure called agglomerative and base up approach called as troublesome strategy [21]-[23]. Dealing with time will turn out to be firm while utilizing this strategy. The factual worth figuring will be simpler while using gridbased strategy [24], [25]. HDFS holds simpler access and extremely more information [26], [27]. Data security is one of the most pressing problems these days. Text-based authentication may be utilized to safeguard data, and a fuzzy rule-based intelligent system can be employed for the authentication process [28]. Given the fact that susceptible software is directly responsible for every security breach, it is desirable to build a descriptive approach that integrates security throughout the requirement phase [29]. Although the objective of usability is to provide simple access to the verified programmed, the primary goal of security is to provide restricted access to the security focused data [30].

\section{ARCHITECTURE}

The definition of software design has been settled (for the time being) with the adoption of the IEEE 1471 standard, which defines software architecture as the fundamental organization of a system embodied in its components, their relationships to each other, and their relationships to the environment. To architect the knowledge and skill set based expert system for online job using clustering and software-agent, it is good choice to follow component-based architecture. With component-based architecture, it is normal that product frameworks can be made and kept up at lower costs and with expanded solidness through reuse of endorsed segments in adaptable programming models. Using component-based architecture one can achieve the goals such as cost reduction, ease of assembly, reusability, customization and flexibility. In Figure 1, shows the different components such as cloud, Job seekers data, clustering agent, optimizing agent and employer database.

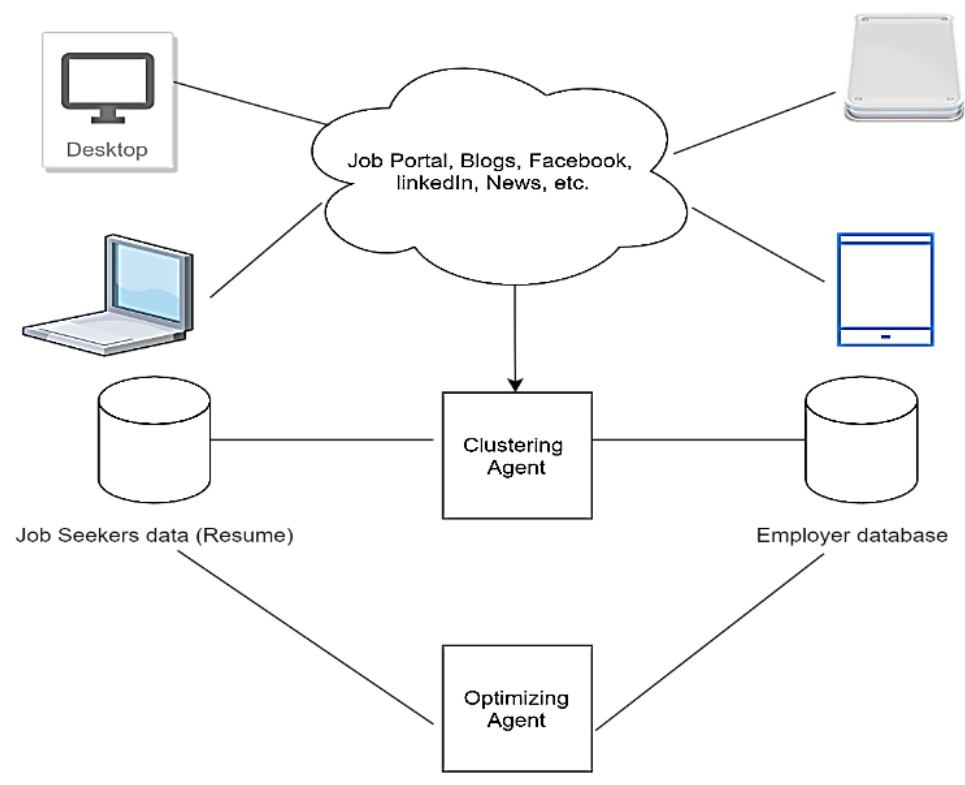

Figure 1. Architecture for knowledge Domains and Skill set based expert systems 


\section{DESCRIPTION OF COMPONENTS}

\subsection{Cloud computing}

The delivery of various services through the internet is known as cloud computing. These resources include data storage, servers, databases, networking, and software, among other tools and applications. As long as an electronic device has internet connectivity, it has access to the data as well as the software applications needed to run it. Examples: Dropbox is a file-sharing and storage service. Microsoft Azure provides cloud based disaster recovery services, as well as hosting and other services. Rack space is a cloud computing company that provides data, protection, and infrastructure services.

\subsection{Job seeker}

Person who is seeking for the job. The below can be requirements of the job seeker from the job portal.

a) Should be able to see all open positions that are scheduled to be filled.

b) Should be able to see the company's information.

c) Should be able to see the vacancy's information.

d) Should be possible to look for positions using the applicant number and the vacancy number.

\subsection{Employer database (recruiting companies)}

a) Should be able to open a new position.

b) Should be able to update any of the vacancy's editable information.

c) The ability to create a new application should be available.

d) Should be able to alter any of the applicant's editable information.

e) Applicant number and vacancy number should be searchable.

f) Assign an applicant to a job opening-the applicant-to-job opening relationship should be many: many.

g) Should be able to organize the interview and input the interviewer's information as well as the date and time.

h) Should be able to reschedule or cancel the interview if necessary.

i) An HR employee cannot 'close' a position that is not theirs.

\subsection{Clustering agent and optimizing agent}

Both the clustering and optimising agents are multi-agent systems. The single administrators who interact with multi-expert structures often exist on a spectrum ranging from heavyweight academic administrators (consistently of "BDI") to lightweight administrators in terms of the administrators' single treatment. Most systems utilize experts from a single circumstance along this reach. We have viably executed a couple of structures where administrators of inside and out various levels of internal refinement associate with one another. Taking into account this experience, we perceive a couple of unmistakable habits by which experts of different kinds can be joined in a single system, offer insights and activities from our experiences.

The role of clustering agent acts as intermediatory between the job seeker and employee database, applies the clustering technique on the curriculum vitae (CV) posted by different job seekers and searches the suitable job in the different employee databases. The role of optimizing agent will provide the optimize skill set for the job seeker to get required skill set for the particular job. The architecture proposed is useful for both job seeker and employer as the clustering agent gets the data ( $\mathrm{CV}$ of the job seeker) from different sources of data through cloud which includes job portals, facebook, linkedIn, clusters them into different categories based on knowledge domains (Job title) and submits to employer database, here the employer database (recruiting company) with the help of optimizing agent gives the optimized skill set needed for particular job title to the job seeker by which the job seeker may enhance his/her skills to apply for the concerned job title.

\section{ALGORITHM}

\section{- Step 1: Job Advertising}

Many companies are using social media for advertising purpose. Some of the social media platforms are Facebook, LinkedIn, and job portals. On the other hand, the companies will also recruit's the employees who apply them directly.

- Step2: CV Screening

The clustering agent which is software agent is used to scan the CV's (resumes) and identify keywords that are correlated with experience, skill set and domain knowledge necessary for the job. This way, the software agent will automatically cluster's the new candidates resume.

- Step 3: Preparing Data

The scanned resumes information will be clustered and stored in the database which will be helpful for the employees. 
- Step 4: Predicting hiring needs

Once the set of resumes have been clustered. The analysis goes a step above the key matching technique. In this stage another software agent is used for optimal search.

- Step 5: Analysis of Resume

The goal software agent here is to analyze the personality of each individual through their resumes and provides a deeper insight into each applicant.

- Step 6: Improve Over Time

The optimize agent will give the individual employer information to improve the skills domain knowledge for the better employment.

\section{CONCLUSION}

The guarantees of component-based architecture for knowledge and skill set based expert system for online job using clustering and software-agent is cost decrease and expanded quality through reuse of demonstrated parts, just as better scalability for programming language. Potential traps for building up partbased programming are execution issues (particular frameworks are regularly less asset efficient than solid frameworks), security, wellbeing, trusted, configuration the executives of complex componentized frameworks. The use of software agents automatically clusters the resumes and give information to the job seekers for enhancing their skills for better job opportunities.

\section{REFERENCES}

[1] E. Faliagka et al., "On-line consistent ranking on e-recruitment: seeking the truthbehind a well-formed CV," The Artificial Intelligence Review, vol. 42, pp. 515-528, 2014.

[2] A. B. Kmail, M. Maree, M. Belkhatir, and S. M. Alhashmi, "An Automatic Online Recruitment System Based on Exploiting Multiple Semantic Resources and Concept-Relatedness Measures," 2015 IEEE 27th International Conference on Tools with Artificial Intelligence (ICTAI), 2015, pp. 620-627, doi: 10.1109/ICTAI.2015.95.

[3] J. Chen, Z. Niu, and H. Fu, "A Novel Knowledge Extraction Framework forResumes Based on Text Classifier," Proceedings of the International Conference on Web-Age Information Management. SpringerInternational Publishing, 2015, pp. 540-543.

[4] C. Hauff and G. Gousios, "Matching GitHub Developer Profiles to Job Advertisements," 2015 IEEE/ACM 12th Working Conference on Mining Software Repositories, 2015, pp. 362-366, doi: 10.1109/MSR.2015.41.

[5] T. Schmitt, P. Caillou, and M. Sebag, "Matching Jobs and Resumes: a DeepCollaborative Filtering Task," Proc. of the 2nd Global Conf. onArtificial Intelligence, 2016, pp. 1-14.

[6] S. Mehta, R. Pimplikar, A. Singh, L. R. Varshney, and K. Visweswariah, "Efficient multifaceted screening of job applicants," Proceedings of the 16th International Conference on Extending Database Technology, 2013, pp. 661-671, doi: $10.1145 / 2452376.2452453$.

[7] International Association of Employment Web Sites (IAEWS). 2021. Accessed: Jun. 20, 2017. [Online]. Avaliable: http://www.icmaonline.org/international-association-of- employment-web-sites

[8] S. Aral and D. Walker, "Creating social contagion through viral product design: A randomized trial of peer influence in networks," Management Science, vol. 57, no. 9, pp. 1623-1639, 2011, doi: 10.1287/mnsc.1110.1421.

[9] D. Centola, "The spread of behavior in an online social network experiment," Science, vol. 329, no. 5996, pp 1194-1197, 2010, doi: 10.1126/science. 1185231 .

[10] S. Leider, M. M. Möbius, T. Rosenblat, and Q.-A. Do, "Directed altruism and enforced reciprocity in social networks," The Quarterly Journal of Economics, vol. 124, no. 4, p. 1815, 2009, doi: 10.1162/qjec.2009.124.4.1815.

[11] A. Katal, M. Wazid, and R. H. Goudar, "Big data: Issues, challenges, tools and Good practices," 2013 Sixth International Conference on Contemporary Computing (IC3), 2013, pp. 404-409, doi: 10.1109/IC3.2013.6612229.

[12] T. Hu, H. Chen, L. Huang, and X. Zhu, "A survey of mass data mining based on cloud-computing," Anti-counterfeiting, Security, and Identification, pp. 1-4, 2012, doi: 10.1109/ICASID.2012.6325353.

[13] J. H. Priyanka and N. Parveen, "Clustering algorithms and storage of clustered data using Hadoop," Journal of green computing. vol. 10, pp. 4744-4751, 2020.

[14] J. S. Bhanu, C. S. Kumar, A. Prakash, and K. V. Raju, "Comprehensive Study on Different Types of Software Agents," International Conference on Intelligent and Smart Computing in Data Analytics, 2021, pp. 255-261.

[15] P. Maes, "Agents that reduce work and information overload," Interactive Technologies, pp. 811-821, 1995.

[16] A. H. Bond, Readings in distributed artificial intelligence, Morgan Kaufmann Publishers, INC, California, 1998.

[17] M. Wooldridge and N. R. Jennings, “Intelligent agents: theory and practice," Cambridge University Press, vol. 10, no. 2, pp. 115-152, 1995.

[18] N. S. Goud and A. Mathur, "A study on conceptual overview of data mining classification and security," Journal of Advanced Research in Dynamical and Control Systems, vol. 10, no. 4, pp, 2050-2054, 2018.

[19] V. Rachapudi, S. V. Suryanarayana, and T. S. Mastan Rao, "Auto-encoder based K-means clustering algorithm," International Journal of Innovative Technology and Exploring Engineering, vol. 8, no. 5, pp. 1223-1226, 2019.

[20] N. Sreeram and K. Satya Prasad, "Efficient map-reduce algorithm for handling very large high dimensional data clustering in heterogeneous multi core environment," Journal of Advanced Research in Dynamical and Control Systems, no. 5, pp. 357-375, 2018.

[21] N. Sirisha and K. V. D. Kiran, “Authorization of data in hadoop using apache sentry,” International Journal of Engineering and Technology, vol. 7, no. 6, pp. 234-236, 2018.

[22] C. Banchhor and N. Srinivasu, "CNB-MRF: Adapting correlative naive bayes classifier and MapReduce framework for big data classification," International Review on Computers and Software, vol. 11, no. 11, pp. 1007-1015, 2016, doi: 10.15866/irecos.v11i11.10116. 
[23] S. Thanekar, K. Subrahmanyam, and A. B. Bagwan, "Big data and MapReduce challenges, opportunities and trends," International Journal of Electrical and Computer Engineering, vol. 6, no. 6, pp. 2911-2919, 2016, doi: 10.11591/ijece.v6i6.10555.

[24] K. Radha and B. T. Rao, "A review on enhancing map reduce performance with data locality in heterogeneous environment," International Journal of Control Theory and Applications, vol. 9, no. 17, pp. 8463-8472, 2016.

[25] V. S. V. Sai, K. Gowthami, N. M. V. R. Shyam, and T. R. Komati, "A systematic survey on dynamic schedulers in Hadoop," Journal of Advanced Research in Dynamical and Control Systems, vol. 9, no. 18, 2017.

[26] A. K. Nikhath and K. Subrahmanyam, "Feature selection, optimization and clustering strategies of text documents," International Journal of Electrical and Computer Engineering, vol. 9, no. 2, pp. 1313-1320, 2019, doi: 10.11591/ijece.v9i2.pp.1313-1320.

[27] L. Greeshma and P. Gera, "Input split frequent pattern tree using mapreduce paradigm in Hadoop," Journal of Theoretical and Applied Information Technology, vol. 84, no. 2, pp. 260-271, 2016.

[28] A. Roy, S. R. Nayak, and R. C. Poonia, "Fuzzy rule based intelligent system for user authentication based on user behavior," Journal of Discrete Mathematical Sciences Cryptography, vol. 23, no. 2, pp. 409-417, 2020, doi: 10.1080/09720529.2020.1728894.

[29] N. Parveen and M. Khaliq, "Difficult on Addressing Security: A Security Requirement Framework," Advances in Intelligent Systems and Computing, pp. 163-172, 2021, doi: 10.1007/978-981-33-6176-8_18.

[30] V. P. Latha, N. Parveen, and Y. Prasanth, "A Fuzzy Approach for Handling Relationship Between Security and Usability Requirements," Advances in Intelligent Systems and Computing, pp. 103-113, 2021.

\section{BIOGRAPHIES OF AUTHORS}
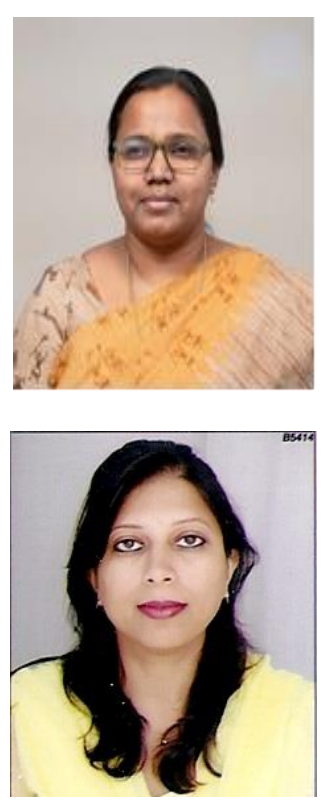

Janampally Himabindu Priyanka (iD SI SC P Research Scholar, Department of Computer Science and Engineering, Koneru Lakshmaiah Education Foundation, vaddeswaram, Guntur, A.P, she is working as assistant professor in CSE Department, Anurag University, Hyderabad.she has total 14 years of teaching experience.she published papers in national and international journals. Her area of interest Big Data, Aritificial Intelligence, IoT. She can be contacted at email: himabindupriyanka1@gmail.com.
Dr. Nikhat Parveen (D) 8 SC $\mathrm{P}$ is Associate Professor in Department of Computer Science and Engineering, Koneru Lakshmaiah Education Foundation, Guntur, A.P. She received B.Sc. (Computer Science) and MCA from Andhra University, Visakhapatnam, India in 2000 and 2003 respectively. She pursued her PhD Degree from Department of Computer Application, Integral University, and Lucknow, India in 2017. She has more than 10 years of teaching experience and 6 years of research experience. Her area of interest is Security Software, Security Testing, Software Engineering, and Requirement Engineering.She is currently working in the area of Soft Computing and Big Data Security Optimization. She has also published \& presented papers in refereed journals and conferences. She is a member of ACM, CSI and IAENG. She can be contacted at email: nikhat0891@gmail.com. 